\title{
Kinetic theory of periodic holes in debunched particle beams
}

\author{
J.-M. Grießmeier and H. Schamel* \\ Physikalisches Institut, Universität Bayreuth, D-95440 Bayreuth, Germany \\ R. Fedele \\ Dipartimento di Scienze Fisiche, Università Federico II and INFN, Complesso Universitario di M. S. Angelo, I-80126 Napoli, Italy
}

(Received 2 November 2001; published 1 February 2002)

\begin{abstract}
A self-consistent theory of periodic hole structures in coasting beams in synchrotrons and storage rings is presented, extending the theory on localized holes. The analysis reveals new intrinsic nonlinear modes which owe their existence to a deficiency of particles trapped in the self-sustained potential well, showing up as notches in the thermal range of the distribution function. It is therefore the full set of Vlasov-Poisson equations which is invoked; linearized treatments as well their nonlinear extensions fundamentally fail to cope with this strongly nonthermodynamic phenomenon. Qualitative agreement with the holes recently found at the CERN proton synchrotron booster is shown.
\end{abstract}

DOI: 10.1103/PhysRevSTAB.5.024201

PACS numbers: 29.27.Bd, 29.20.Lq, 52.35.Sb

\section{INTRODUCTION}

Recently, progress was made in the analysis of electrostatic structures [1-5] with the aim of explaining longitudinal structures observed in coasting particle beams of circular accelerators $[6,7]$ as well as in numerical experiments [7-9]. Attention was focused on a unique, in a sense novel, feature of these structures, namely their nonlinear nonhydrodynamic character. Modes of this type were shown to owe their existence to the trapping of particles in the self-sustained potential trough. As there is no threshold in wave amplitude, this process is nonlinear from the outset in a collision-free environment. In other words, eddies in phase space as representatives of trapped particles can be arbitrarily small. Nevertheless they do play a fundamental role in the description of the collective dynamics of an unbunched particle beam. They are due to the wave-particle resonance between particles and fluctuations mainly in the thermal range of the momentum distribution for which linearized wave solutions $[10,11]$ are inapplicable no matter how small the excitation level. This intrinsic nonlinearity implies that a particle beam of sufficiently high brightness representing a more or less collisionless one-component plasma has to be described by the fully nonlinear set of the Vlasov equation coupled selfconsistently to a Poisson-like elliptic equation. Methods and theorems developed in plasma physics $[12,13]$ can, therefore, be directly applied to beam physics.

The goal of the present work is to extend the existing theory of solitary hole structures [1-5] to periodic hole structures. We also attempt to explain some features of the periodic longitudinal structures recently observed at the CERN proton synchrotron booster [9].

*Electronic address: hans.schamel@uni-bayreuth.de
The paper is organized as follows. In Sec. II we present the governing equations. In Sec. III a solution for the Vlasov equation is derived, and the solutions for the wave potential are obtained in Sec. IV. Section V is devoted to the periodicity condition. Some explicit solutions are given in Sec. VI, and Sec. VII terminates the paper with some concluding remarks.

\section{BASIC EQUATIONS}

The governing equations valid for highly relativistic beams $\left[\gamma=\left(1-v^{2} / c^{2}\right)^{-1 / 2} \gg 1\right]$ were derived in [5] [see Eqs. (9) and (10) therein] to which we refer also with respect to the definitions and normalizations. Formulated in the frame of reference moving with the nominal beam velocity $v$, they read

$$
\begin{gathered}
\left(\partial_{t}+u \partial_{z}-\epsilon \partial_{u}\right) f=0, \\
(1-L) \epsilon^{\prime \prime}+R \epsilon^{\prime}-\mu \epsilon=\alpha\left[R \lambda_{1}+\left(g_{0}-L\right) \lambda_{1}^{\prime}\right] .
\end{gathered}
$$

Equation (1) is the Vlasov equation for the beam distribution function $f(z, u, t)$. It is assumed that the beam can be treated nonrelativistically in the frame of reference of the synchronous particle along the design orbit (comoving frame). Equation (1) is coupled to the electric field $\epsilon$ via the generalized version of Poisson's equation (2). The derivation of Eq. (2) can be found in the appendix of Ref. [5]. It assumes a step-function density profile for the beam ions in the directions perpendicular to its axis of propagation. The derivation takes into account $\mathcal{O}\left(\gamma^{-2}\right)$ effects arising from the longitudinal field, representing an electromagnetic correction. The parameters $R$ and $L$ are measures for the resistivity and inductivity of the surrounding wall, respectively. The perturbation of the line density $\lambda_{1}$ is given by

(C) 2002 The American Physical Society

024201-1 


$$
\lambda_{1}=\int_{-\infty}^{+\infty} f(z, u) d u-1 .
$$

The remaining parameters in (2) are the geometry factor $g_{0}>0$ and the two machine parameters $\alpha$ and $\mu$. $\alpha$ is proportional to $\eta N$ where $\eta$ is the slip factor and $N$ is the total number of beam particles. According to [5], $\alpha$ carries the sign of the slip factor. $\mu$ is positive and proportional to $\left(\gamma R_{0}\right)^{2}$ where $R_{0}$ is the large radius of the synchrotron. The latter two are typically large quantities as $\gamma$ was assumed to be large.

Equation (2) has two well-known limits: the resistive limit, $R \gg 1$, for which

$$
\epsilon^{\prime}=\alpha \lambda_{1},
$$

and the purely reactive limit, $R=0$, for which we get the following expression:

$$
(1-L) \epsilon^{\prime \prime}-\mu \epsilon=\alpha\left(g_{0}-L\right) \lambda_{1}^{\prime} .
$$

If one assumes $\alpha$ and $\mu$ to be very large, Eq. (4b) simplifies to

$$
\epsilon=-\frac{\alpha\left(g_{0}-L\right)}{\mu} \lambda_{1}^{\prime} .
$$

This coincides with the expression given in [14]. The first term on the right-hand side represents the capacitive spacecharge effect, and the second term represents the inductive contribution of the imaginary impedance.

\section{SOLUTION OF THE VLASOV EQUATION}

To solve the coupled system of Eqs. (1) and (2) we make use of the "potential method" derived by Schamel [15]. It is used in plasma physics to find steady-state solutions in an appropriate frame of reference, namely the frame propagating with the wave structure. It consists of first solving the Vlasov equation to find the distribution function; in a second step the associate potential structure is determined. It was shown earlier [15] that this method yields physical solutions in contrast to the so-called BernsteinGreene-Kruskal method [16].

To solve the Vlasov equation (1) we first perform a Galilei transformation to a frame moving with $\Delta u$, the phase velocity of the structure in the comoving frame. We assume that the particle distribution of the unperturbed system is given by a Maxwellian in the latter frame, which implies that in the new frame of reference (which we call wave frame) the unperturbed beam is described by a shifted Maxwellian:

$$
f(u)=\frac{1}{\sqrt{2 \pi}} \exp \left[-\frac{1}{2}(u+\Delta u)^{2}\right] .
$$

Note that at this stage $\Delta u$ is an unknown quantity, and that its determination is part of the final solution.

Since (1) also holds in the wave frame, we can find a stationary solution $\left(\partial_{t}=0\right)$ for the perturbed beam by setting

$$
f(z, u)= \begin{cases}\frac{1+K}{\sqrt{2 \pi}} \exp \left\{-\frac{1}{2}\left[\sigma \sqrt{u^{2}-2 \Phi(z)}+\Delta u\right]^{2}\right\} & \text { for } E \geq 0 \\ \frac{1+K}{\sqrt{2 \pi}} \exp \left\{-\frac{1}{2}\left[\beta\left(u^{2}-2 \Phi(z)\right)+\Delta u^{2}\right]\right\} & \text { for } E<0\end{cases}
$$

where $\Phi(z)$ represents the electrostatic potential defined by $\epsilon=-\Phi^{\prime}(z)$ and

$$
E=\frac{1}{2} u^{2}-\Phi(z)
$$

is the single particle energy. The distribution function depends on two constants of the motion, namely $E$ and the sign of the velocity of an untrapped particle $\sigma=\operatorname{sgn}(u)$. Without loss of generality, a non-negative potential is assumed: $0 \leq \Phi(z) \leq \Psi$, where we call $\Psi$ the amplitude of the wave structure. Thus all particles with $E<0$ are trapped in the potential structure, and the lower part of Eq. (6) describes trapped particles. Note that the particle distribution (6) is continuous in momentum space and reduces to (5) in the unperturbed limit, $\Psi \rightarrow 0$, as we require that $K$ is vanishing for $\Psi \rightarrow 0$. The parameter $\beta$ controls the occupation of trapped particle orbits. We call $\beta$ the trapping parameter; it turns out to be a decisive parameter for the whole theory. If it is negative, the trapped range is less densely occupied than the neighboring free regions in momentum space, and the distribution function has a notch.

By integrating Eq. (6) over the velocity $u$ we can calculate the perturbation of the line density $\lambda_{1}$; see Eq. (3). $\lambda_{1}$ now becomes a functional of $\Phi$. Performing a Taylor expansion in terms of $\Phi$ we finally get an expression for the perturbation of the line density [17] in the small amplitude limit $\Psi \ll 1$ :

$$
\begin{aligned}
\lambda_{1}= & K-\frac{1}{2} Z_{r}^{\prime}\left(\frac{\Delta u}{\sqrt{2}}\right)(1+K) \Phi \\
& -\frac{4}{3} b(\beta, \Delta u)(1+K) \Phi^{3 / 2} \\
& +\frac{1}{16} Z_{r}^{\prime \prime \prime}\left(\frac{\Delta u}{\sqrt{2}}\right) \Phi^{2} .
\end{aligned}
$$

Expression (8) is valid up to $\mathcal{O}\left(\Psi^{2}\right) . Z_{r}(x)$ refers to the real part of the so-called plasma dispersion function [18] given by

$$
Z(x)=\exp \left(-x^{2}\right)\left[i \sqrt{\pi}-2 \int_{0}^{x} d t \exp \left(t^{2}\right)\right]
$$

for real arguments. The function $b(\beta, \Delta u)$ is defined as

$$
b(\beta, \Delta u)=\frac{1}{\sqrt{\pi}}\left(1-\beta-\Delta u^{2}\right) \exp \left(-\frac{\Delta u^{2}}{2}\right) .
$$


We will allow $b(\beta, \Delta u)$ to be of the order of $\mathcal{O}\left(\Psi^{-1 / 2}\right)$. The parameter $K$ is required for periodic solutions; $K=0$ in the solitary wave limit [1-5].

\section{SOLUTION FOR THE WAVE POTENTIAL}

As in Refs. [1-5], we restrict the following analysis to the two limiting cases: (i) the resistive limit $(R \gg 1)$

$$
\Phi^{\prime \prime}(z)=-\alpha \lambda_{1},
$$

and (ii) the purely reactive limit $(R=0)$

$$
\Phi^{\prime \prime}(z)=-\bar{\alpha} \lambda_{1}+\bar{\mu} \Phi,
$$

which follow from Eqs. (4a) and (4b), respectively. In (11b) we introduced

$$
\bar{\alpha}=\alpha \frac{g_{0}-L}{1-L} \quad \text { and } \quad \bar{\mu}=\frac{\mu}{1-L} .
$$

$\alpha$ is negative as long as the beam energy $E_{0}=m \gamma c^{2}$ is lower than the transition energy [14]. Where not stated otherwise, only this case will be considered further.

Inserting $\lambda_{1}$ from (8) into (11), we obtain an equation of the type

$$
\Phi^{\prime \prime}(z)=Z+A \Phi+B \Phi^{3 / 2}+C \Phi^{2}+\cdots,
$$

in which the constants $Z, A, B$, and $C$ still depend on the case considered; for $R \gg 1$ we have, for example, $Z \equiv Z_{\infty}=-\alpha K$. For $R=0, B$ is given by $B \equiv B_{0}=$ $4 / 3 \bar{\alpha} b(\beta, \Delta u)$, etc.

The simple form (13) allows a direct integration

$$
\frac{\Phi^{\prime}(z)^{2}}{2}+V(\Phi)=0
$$

where $-V^{\prime}(\Phi)$ is given by the right-hand side of (13). In (14) we used $V(0)=0$ which follows from the minimum of the potential at $\Phi=0$. With this, $-V(\Phi)$ becomes

$$
-V(\Phi)=Z \Phi+\frac{1}{2} A \Phi^{2}+\frac{2}{5} B \Phi^{5 / 2}+\frac{1}{3} C \Phi^{3}+\cdots .
$$

Together with (14) the form of $V(\Phi)$ uniquely defines the wave structure. For a periodic structure we must require $V^{\prime}(0)<0$, from which follows $Z>0$. In the resistive case we thus get $K>0$ (as $\alpha<0$ ), and define

$$
K \equiv K_{\infty}=\frac{k_{0}^{2} \Psi}{2}
$$

which vanishes for $\Psi \rightarrow 0$ and is defined as such to allow closest contact to linearized theory (namely to the Vlasov solution; see Ref. [13]). In the reactive case, we have to use $Z_{0}=-\bar{\alpha} K_{0}>0$ and get

$$
K \equiv K_{0}=-\operatorname{sgn}(\bar{\alpha}) \frac{k_{0}^{2} \Psi}{2}
$$

where the definition of $K_{0}$ depends not only on $\alpha$ but also on the beam parameters $g_{0}$ and $L$ as well.

The necessary condition $V(\Psi)=0$, which represents the maximum of the potential, becomes

$$
Z+\frac{1}{2} A \Psi+\frac{2}{5} B \Psi^{3 / 2}+\frac{1}{3} C \Psi^{2}=0 .
$$

This relation is called the nonlinear dispersion relation (NDR) since it determines $\Delta u$ in terms of the other parameters $(\beta, \Psi ; \alpha, \mu, \ldots)$.

We now concentrate on conditions for which the last term in (13), (15), and (18) is negligible, demanding $|B| \gg$ $|C| \sqrt{\Psi}$. Keeping $K \ll 1$, we then get for the NDR in the resistive case

$$
k_{0}^{2}-\frac{1}{2} Z_{r}^{\prime}\left(\frac{\Delta u}{\sqrt{2}}\right)=\frac{16}{15} b(\beta, \Delta u) \sqrt{\Psi} .
$$

This expression has already been obtained in the past in several publications (see Ref. [13] and the references therein).

Making use of (19), the reduced form of (15) can be rewritten as

$$
\begin{aligned}
-V(\Phi)=-\alpha[ & \frac{k_{0}^{2}}{2} \Phi(\Psi-\Phi) \\
& \left.+\frac{8}{15} b(\beta, \Delta u) \Phi^{2}(\sqrt{\Psi}-\sqrt{\Phi})\right] .
\end{aligned}
$$

This expression coincides with the previous expressions for the plasma case (e.g., in [17]), except for the positive factor $-\alpha$ on the right-hand side of (20). It can be replaced by unity if the longitudinal coordinate $z$ is rescaled $(\sqrt{-\alpha} z \rightarrow z)$. Note that $k_{0}=0$ in (19) and (20) represents the solitary wave limit treated previously [1-5]. On the other hand, in the space-charge dominated case $R=L=0$ (which is realized in the experiment of [9]), we get two expressions for the NDR and for $V(\Phi)$ which are identical with Eqs. (19) and (20), respectively, if $k_{0}^{2}$ is replaced by $\hat{k}_{0}^{2} \equiv k_{0}^{2}-\mu /\left(\alpha g_{0}\right)>0$ for $\alpha<0$ (below transition energy) and by $-\tilde{k}_{0}^{2} \equiv-\left[k_{0}^{2}+\mu /\left(\alpha g_{0}\right)\right]<0$ for $\alpha>0$ (above transition energy). The potential $\Phi(z)$ given by (14) and (20) can be taken from Ref. [17].

\section{PERIODICITY CONDITION}

The periodic nonlinear structure we are looking for must fit into the machine, i.e., it must satisfy the periodicity condition

$$
\Phi(z+1)=\Phi(z) .
$$

Note that the dimensionless coordinate $z$ is defined as the dimensional coordinate normalized by $2 \pi R_{0}$, the circumference of the machine. If $2 L$ describes the dimensionless periodicity length of the structure, which can be found in accordance with the final quadrature of (14) using (20) (see Refs. [15,17]), Eq. (21) implies

$$
2 L n=1 \quad \text { with } n=1,2,3, \ldots .
$$


Following the notation of $[15,17]$ we now introduce the combined parameter

$$
\hat{L}:=\frac{15 k_{0}^{2}}{64 b(\beta, \Delta u) \sqrt{\Psi}} .
$$

It will be shown later that this parameter controls the harmonic wave spectrum of the periodic solution in the resistive case. Note that $\hat{L}$ is not directly related to the periodicity length $L$. Three regions of wave solutions can be distinguished in which $\Phi(z)$ can be expressed by Jacobian elliptic functions [15,17],

$$
\begin{aligned}
\text { (i) } & \hat{L}<-\frac{1}{8} \\
\text { (ii) } & 0 \leq \hat{L} \leq 1 \\
\text { (iii) } & 1<\hat{L}
\end{aligned}
$$

Applying (22) to the solution of $[15,17]$ for each region we get the following periodicity conditions:

$$
\begin{aligned}
n F\left(\arcsin \sqrt{\frac{1-K_{1} / K_{2}}{1-K_{1}}} \mid m_{1}\right) & =\frac{1}{2} \sqrt{-\alpha} \sqrt{1-\frac{K_{1}}{K_{2}}} \sqrt{-\frac{K_{2} b \sqrt{\Psi}}{15}}, \\
n F\left(\arccos \frac{K_{4}-1}{K_{4}+1} \mid m_{2}\right) & =\sqrt{-\alpha} \sqrt{\frac{K_{4} b \sqrt{\Psi}}{15}}, \\
n F\left(\arcsin \frac{1}{\sqrt{K_{5}}} \mid m_{3}\right) & =\frac{1}{2} \sqrt{-\alpha} \sqrt{\frac{K_{6} b \sqrt{\Psi}}{15}}
\end{aligned}
$$

where $F(u \mid m)$ denotes the elliptic integral of the first kind as defined in [19], $b$ stands for $b(\beta, \Delta u)$, and the $K_{i}$ 's and $m_{i}$ 's are given by

$$
\begin{aligned}
& K_{1}=1+2 \hat{L}-2 \sqrt{\hat{L}(\hat{L}-1)} \equiv K_{5}, \\
& K_{2}=1+2 \hat{L}+2 \sqrt{\hat{L}(\hat{L}-1)} \equiv K_{6}, \\
& K_{3}=1+2 \hat{L}, \\
& K_{4}=1-\sqrt{1+8 \hat{L}}, \\
& m_{1}=\frac{K_{2}}{K_{2}-K_{1}}, \\
& m_{2}=\frac{1}{2}\left(1+K_{3} / K_{4}\right), \\
& m_{3}=K_{5} / K_{6} .
\end{aligned}
$$

Through the $K_{i}$ 's each periodicity condition in (25) depends on $\hat{L}$ which itself depends on $k_{0}, \Psi$, and $b(\beta, \Delta u)$. They are therefore implicit equations which have to be satisfied by the parameters. In addition, the NDR (19) has to be satisfied. It can be rewritten as

$$
k_{0}^{2}-\frac{1}{2} Z_{r}^{\prime}\left(\frac{\Delta u}{\sqrt{2}}\right)=\frac{k_{0}^{2}}{4 \hat{L}} .
$$

We emphasize that (27) admits standing wave solutions $(\Delta u=0)$. In that case, $Z_{r}^{\prime}\left(\frac{\Delta u}{\sqrt{2}}\right)=-2$, and $\hat{L}$ is given by $4 \hat{L}=k_{0}^{2} /\left(k_{0}^{2}+1\right)$. Hence, for any value of $\hat{L}$ in the interval $0 \leq \hat{L} \leq 1 / 4$ standing (periodic or solitary) wave solutions are possible.

\section{SOME EXPLICIT SOLUTIONS}

The two conditions (25) and (27) are now investigated for some special cases. For $m=0$ and $m=1$ the elliptic integral simplifies as follows:

$$
\begin{aligned}
& F(u \mid 0)=u, \\
& F(u \mid 1)=\ln \left[\tan \left(\frac{\pi}{4}+\frac{u}{2}\right)\right]=\ln (\sec u+\tan u) .
\end{aligned}
$$

\section{A. $\hat{L} \rightarrow-\infty$ (harmonic potential)}

For this limit, which can be achieved by letting $b(\beta, \Delta u) \rightarrow 0^{-}$, we find $K_{1} \rightarrow 4 \hat{L}, K_{2} \rightarrow 2$, and hence $m_{1} \rightarrow 0 . b(\beta, \Delta u)=0$ in (20) yields a harmonic potential $\Phi(z)$ given by

$$
\Phi(z)=\frac{\Psi}{2}\left[1+\cos \left(\sqrt{-\alpha} k_{0} z\right)\right]=\Psi \cos ^{2}\left(\frac{\sqrt{-\alpha} k_{0} z}{2}\right) .
$$

The periodicity condition (25a) and the NDR (27) simplify to

$$
\begin{aligned}
& k_{0}^{2}=\frac{(2 \pi n)^{2}}{-\alpha}, \\
& k_{0}^{2}=\frac{1}{2} Z_{r}^{\prime}\left(\frac{\Delta u}{\sqrt{2}}\right),
\end{aligned}
$$

respectively. Since $Z_{r}^{\prime}(x)$ is positive in the region $0.924<$ $x$ with a maximum of 0.285 at $x=1.50$, we see that a solution is possible if $k_{0}^{2}<0.285,-\alpha>138.64$, and $\Delta u>1.307$. For a given $\alpha$, the periodicity parameter $n$ (number of potential maxima) may not be too large. Since $\alpha$ is proportional to the number of beam particles $N$, the condition for $-\alpha$ can be satisfied easily. From the definition (10) it follows that $\beta$ has to be negative, which implies a depression of the distribution function in the resonant region. 


\section{B. $\hat{\boldsymbol{L}} \rightarrow \mathbf{0}^{+}$(solitary potential)}

This limit requires $k_{0} \rightarrow 0$. Using (20), the potential structure becomes [1]

$$
\Phi(z)=\Psi \operatorname{sech}^{4} \sqrt{\frac{-\alpha b \sqrt{\Psi}}{15}} z .
$$

With $K_{3} \rightarrow 1, K_{4} \rightarrow 1$, and $m_{2} \rightarrow 1$, the periodicity condition $(25 b)$ is found to be

$$
n \ln \left[\tan \left(\frac{\pi}{2}\right)\right]=\sqrt{\frac{-\alpha b \sqrt{\Psi}}{15}},
$$

which is infinite on the left-hand side and, strictly speaking, not solvable. However, since the solitary wave structure rapidly decays for distances larger than $\Delta:=15\left[-\alpha b\left(\beta, \hat{v}_{d}\right) \sqrt{\Psi}\right]^{-1}$, which in turn can be very small due to $|\alpha|^{-1} \propto N^{-1}$, many solitary structures can coexist in the device with no mutual interaction. Thus the periodicity condition (32) loses relevance in real devices if $N \gg 1$, which is usually the case.

\section{C. $\hat{L} \rightarrow 1^{-}$(anharmonic potential)}

In this limit we get from (26): $K_{3} \rightarrow 3, K_{4} \rightarrow 3$, and $m_{2} \rightarrow 1$. The potential is then given by

$$
\Phi(z)=\Psi\left[1-3 \tanh ^{2}\left(\sqrt{\frac{3}{64}(-\alpha)} k_{0} z\right)\right]^{2} .
$$

Equation (33) is valid for $-L \leq z \leq+L$, where $L$ is given by $L / 4=\left[3(-\alpha) k_{0}^{2}\right]^{-1 / 2} \ln (2+\sqrt{3})$. Outside this range $\Phi(z)$ is found by periodic continuation. Using (23), the periodicity condition $(25 \mathrm{~b})$ becomes

$$
n \ln (2+\sqrt{3})=\sqrt{\frac{-\alpha b \sqrt{\Psi}}{5}}=\sqrt{\frac{3(-\alpha) k_{0}^{2}}{64}},
$$

which has to be satisfied in conjunction with the NDR (27), which simplifies to

$$
\frac{1}{2} Z_{r}^{\prime}\left(\frac{\Delta u}{\sqrt{2}}\right)=\frac{3}{4} k_{0}^{2} .
$$

As in the harmonic case (Sec. VI. A), a solution can be found provided that $-\alpha$ is not too small. The necessary conditions are $-\alpha>97.45, k_{0}^{2}<0.38$, and the wave velocity has to satisfy $\Delta u>1.307$ such that $Z_{r}^{\prime}\left(\frac{\Delta u}{\sqrt{2}}\right)$ is positive. For a given $\alpha$, here again $n$ must not be too large. For given values $-\alpha, \Psi, k_{0}$, and $\Delta u>1.307$, and using the condition $b(\beta, \Delta u) \sqrt{\Psi}=\frac{15}{64} k_{0}^{2}$, which results from $\hat{L}=1$, we find from definition (10) that $\beta$ must be sufficiently negative to meet all conditions. Thus, the distribution function again has to be depressed, i.e., holelike, in the resonant regions.

\section{Other periodic solutions for the resistive case}

It can be easily checked that approaching $\hat{L} \rightarrow+\infty$ and $\hat{L} \rightarrow 1^{+}$in Eq. (25c) yields the same result as in cases VIA and VIC, respectively. Other members of the class of periodic solutions can be found if $\hat{L}$ is allowed to take values differing from those cases mentioned above. In general, this requires a numerical evaluation of the two constraining conditions, namely the periodicity condition (25) and the nonlinear dispersion relation (27) to determine the parameters $k_{0}, \Delta u$, and $\beta$.

\section{E. Space-charge dominated case}

Until now we considered only the resistive case. To treat the case realized in the experiments of [9], namely that of a purely space-charge dominated beam below transition energy, $k_{0}^{2}$ in Eqs. (19), (20), and (23) has to be replaced by $\hat{k}_{0}^{2}=k_{0}^{2}-\mu /\left(\alpha g_{0}\right)$, as was mentioned previously.

In the harmonic limit $\hat{L} \rightarrow-\infty$ the existence conditions are that of (30) with $k_{0}^{2}$ replaced by $\hat{k}_{0}^{2}$. The conditions therefore become

$$
\begin{aligned}
& k_{0}^{2}-\frac{\mu}{\alpha g_{0}}=\frac{(2 \pi n)^{2}}{-\alpha}>0, \\
& k_{0}^{2}-\frac{\mu}{\alpha g_{0}}=\frac{1}{2} Z_{r}^{\prime}\left(\frac{\Delta u}{\sqrt{2}}\right)>0 .
\end{aligned}
$$

Again, $\Delta u$ must exceed 1.307, i.e., nonpropagating structures (with $\Delta u=0$ ) do not exist in this limit. From $k_{0}^{2}-\mu / \alpha g_{0}<0.285$ it follows that the machine parameters have to satisfy the condition

$$
\frac{\mu}{\alpha g_{0}}=\frac{8 \pi^{2} \epsilon_{0} m \gamma^{3} R_{0} v^{2}}{|\eta| q^{2} g_{0} N}<0.285,
$$

which can be satisfied easily if the total number of beam particles is sufficiently large $\left(N \sim \mathcal{O}\left(10^{12}\right)\right.$ in Ref. [9]). See Ref. [5] for the definitions of $\mu$ and $\alpha$.

As mentioned earlier, the observation of a standing periodic wave structure under space-charge dominated conditions as in Ref. [9] can be explained by assuming $0 \leq \hat{L} \leq 1 / 4$, which is a subset of case (ii) in (24), subject to the periodicity condition $(25 \mathrm{~b})$. Note that in this situation $\hat{L}$ is given by $4 \hat{L}=\hat{k}_{0}^{2} /\left(\hat{k}_{0}^{2}+1\right)$.

We conclude that periodic holes, as a solution of the full nonlinear set of Vlasov-Poisson equations, can exist under a variety of experimental conditions and hence are ubiquitous in such systems. The observation of "phase space voids introduced by empty rf buckets that do not debunch" in Ref. [9] is then not surprising as it reflects a natural event in debunched beams.

However, some caution must be given. The present analysis was based on small holes only $(\Psi \ll 1)$, which allows analytic evaluation. In general, $\Psi$ can be arbitrarily large representing finite amplitude holes $[12,20]$. Then the existence conditions have to be obtained numerically.

\section{CONCLUDING REMARKS}

In this paper, the theory of localized holes in debunched beams has been generalized to periodic wave trains. Conditions for the existence of stationary and self-consistent 
solutions have been derived. As was shown, these solutions require a nonlinear analysis of the Vlasov-Poison system and hence lie outside the realm of linear wave theories. The question of stability and longevity of hole structures as studied numerically in [9] (whether holes coalesce, split, or remain essentially unchanged) is a different issue and will be investigated in forthcoming publications. We mention only that a modified Kortewegde Vries equation - the so-called Schamel equation - can be derived [21] admitting our phase space vortices as special solutions. The complete integrability of this equation is, however, doubtful [22-24] and hence inelastic collisions such as coalescence can be expected to be involved in the dynamical evolution.

\section{ACKNOWLEDGMENTS}

This work was supported by CRUI (Conferenza dei Rettori delle Università Italiane) and DAAD (Deutscher Akademischer Austauschdienst) within the research program "Vigoni" between the University Federico II of Napoli and the University of Bayreuth (project "Nonlinear Phenomena, Instability and Macroscopic Coherence in Charged-Partice Beam Dynamics").

[1] H. Schamel, Phys. Rev. Lett. 79, 2811 (1997).

[2] H. Schamel, Phys. Scr. T75, 23 (1998).

[3] H. Schamel, in Nonlinear and Stochastic Beam Dynamics in Accelerators: A Challenge to Theoretical and Computational Physics, Lüneburg, 1997 (DESY, Hamburg, Germany, 1998), pp. 224-235.
[4] H. Schamel and R. Fedele, in Proceedings of the 7th European Particle Accelerator Conference, Vienna, 2000, http://accelconf.web.cern.ch/Accelconf/e00/index.html.

[5] H. Schamel and R. Fedele, Phys. Plasmas 7, 3421 (2000).

[6] P. L. Colestock and L. K. Spentzouris, in The Future of Accelerator Physics: The Tamura Symposium, edited by T. Tajima, AIP Conf. Proc. No. 356 (AIP, New York, 1996).

[7] O. Boine-Frankenheim, I. Hofmann, and G. Rumolo, Phys. Rev. Lett. 82, 3256 (1999).

[8] I. Hofmann, Laser Part. Beams 3, 1 (1985).

[9] S. Koscielniak, S. Hancock, and M. Lindroos, Phys. Rev. ST Accel. Beams 4, 044201 (2001).

[10] A. Ruggiero and V. Vaccaro, CERN Report No. CERNISR/TH/68-33, 1968.

[11] E. Keil and W. Schnell, CERN Report No. ISR-THRF/69-48, 1969.

[12] H. Schamel, Phys. Rep. 140, 161 (1986).

[13] H. Schamel, Phys. Plasmas 7, 4831 (2000).

[14] A. Hofmann, in Proceedings of the International School of Particle Accelerators, Erice, Italy, edited by H. W. Blewett (CERN, Geneva, 1977), pp. 139-174.

[15] H. Schamel, Plasma Phys. 14, 905 (1972).

[16] I. B. Bernstein, J. M. Greene, and M. D. Kruskal, Phys. Rev. 108, 546 (1957).

[17] J. Korn and H. Schamel, J. Plasma Phys. 56, 307 (1996).

[18] B. D. Fried and S. D. Conte, The Plasma Dispersion Function (Academic Press, New York, 1961).

[19] Handbook of Mathematical Functions, edited by $\mathrm{N}$. Abramowitz and I. A. Stegun (Dover, New York, 1972).

[20] S. Bujarbarua and H. Schamel, J. Plasma Phys. 25, 515 (1981).

[21] H. Schamel, J. Plasma Phys. 9, 377 (1973).

[22] M. W. Coffey, J. Phys. A 24, L1345 (1991).

[23] A. Ramani and B. Grammaticos, J. Phys. A 24, 1969 (1991).

[24] F. Verheest and W. Hereman, Phys. Scr. 50, 611 (1994). 